\title{
A case of COVID-19 complicated by Klebsiella pneumoniae, Cytomegalovirus, Aspergillus and Zygomycete infections
}

\author{
Mihan Pourabdollah ${ }^{1}$, Atousa Hakamifard ${ }^{1}$, Zahra Abtahian ${ }^{1}$, Arefeh Mirahmadian ${ }^{1}$, \\ Mohammad Rahdar ${ }^{1}$, Mojtaba Mokhber Dezfuli ${ }^{1}$, and Payam Tabarsi ${ }^{1}$ \\ ${ }^{1}$ Shahid Beheshti University of Medical Sciences
}

January 4, 2022

\begin{abstract}
Co-infection between SARS-CoV-2 and other pathogens have become a serious threat. There are reports of fungal, bacterial and viral co-infections with SARS-COV-2. Herein, we report the unusual case of concomitant aspergillosis, mucormycosis, cytomegalovirus pneumonia and also klebsiella pneumoniae empyema as the complication of COVID-19 infection.
\end{abstract}

A case of COVID-19 complicated by Klebsiella pneumoniae, Cytomegalovirus, Aspergillus and Zygomycete infections

\begin{abstract}
Co-infection between SARS-CoV-2 and other pathogens have become a serious threat. There are reports of fungal, bacterial and viral co-infections with SARS-COV-2. Herein, we report the unusual case of concomitant aspergillosis, mucormycosis, cytomegalovirus pneumonia and also klebsiella pneumoniae empyema as the complication of COVID-19 infection.
\end{abstract}

Keywords: CMV, Klebsiella pneumoniae, mixed infection, SARS-COV-2, mucormycosis, aspergillosis

Key messages:

Secondary infections or co-infections are identified between SARS-CoV-2 and other pathogens. This entity have become another serious threat in the treatment of patients with COVID-19 infection which should not be neglected.

\section{Introduction}

Secondary infections or co-infections are commonly identified in severe influenza and also other severe respiratory viral infections with high mortality and morbidity rate [1]. Co-infection between SARS-CoV-2 and other pathogens have become another serious threat in the treatment of patients which should not be neglected [2]. These co-pathogens are including bacteria, such as Streptococcus pneumoniae andStaphylococcus aureus, fungal pathogens includingAspergillus or Mucorales species and also viruses such as influenza, and rhinovirus/enterovirus [3]. Herein we report the first case of concomitant aspergillosis, mucormycosis, cytomegalovirus (CMV) pneumonia and also klebsiella pneumoniae empyema as the complication of COVID-19 infection who had a favorable clinical outcome.

\section{Case presentation}

A 68-year-old diabetic man was presented to the emergency department with a five days history of fever $\left(38.7^{\circ} \mathrm{C}\right)$, dry cough, and dyspnea ( $\mathrm{SpO} 2$ was $90 \%$ - room air). He was on oral hypoglycemic agent (metformin $500 \mathrm{mg}$ every 12 hours) for several years. Laboratory studies showed elevated $\mathrm{C}$ reactive protein 
(120 mg/dl), and RT-PCR confirmed SARS-COV-2 diagnosis. High-resolution chest CT scan obtained at admission was consistent with COVID-19 pneumonia. The patient received remdesivir and dexamethasone and was discharged after 9 days with improvement of signs and symptoms. After 12 days the patient was re-admitted because of severe dyspnea and fever. Chest CT scan revealed left sided pleural effusion and the chest tube was inserted and discharged after seven days with improvement of dyspnea. After 10 days the patient was referred to our center for the first time, because of malaise, fever, chills and purulent discharge at previous chest tube excite site.

At admission the patient was febrile and the vital signs were as following: PR: 110 beats/min, RR: 25 breath/min T: 38.5 and BP: 120/90 mmHg. The initial laboratory data were as following: white blood cell count (WBC): 4900/ $\mathrm{mm}^{3}$ (neutrophils: 83\%, lymphocytes: $8 \%$ ), hemoglobin (Hb): $10.2 \mathrm{gr} / \mathrm{dl}$, platelets (PLT): 250,000/ $\mathrm{mm}^{3}$, blood sugar: 187 and creatinine: $1.1 \mathrm{mg} / \mathrm{dl}$. Also COVID-19 PCR was reported positive. Pleural fluid was sent for analysis, bacterial and fungal smear and culture. Blood cultures were negative. The pleural fluid analysis were as following: turbid appearance, white blood cell $>500,000$, polymorphonuclear $90 \%$, lactate dehydrogenase $(\mathrm{LDH}) 26,448 \mathrm{U} / \mathrm{L}$, sugar $<10 \mathrm{mg} / \mathrm{dl}$, protein $2.9 \mathrm{gr} / \mathrm{dl}$, albumin $1.9 \mathrm{gr} / \mathrm{dl}$. Serum LDH level, total protein and albumin were $523 \mathrm{U} / \mathrm{L}, 5.1 \mathrm{gr} / \mathrm{dl}$ and $3.2 \mathrm{gr} / \mathrm{dl}$ respectively, indicative of empyema and chest tube was inserted.

Imipenem $500 \mathrm{mg}$ every 6 hours and vancomycin 1 gr every 12 hours were started as empiric treatment. The result of culture was reportedklebsiella pneumoniae which was sensitive to meropenem and also imipenem. The vancomycin was discontinued and imipenem with amikacin was continued. The patient presented extensive subcutaneous emphysema in both sides and left chest tube was inserted (Figure 1). After several days the left sided chest tube was came out. According to the right sided empyema and no significant improvement, the patient underwent right thoracotomy with decortication. In addition, right upper lobe and right lower lobe wedge resection, hilar lymph node biopsy and partial pleural peel resection was performed. After the operation, the whole lung was expanded and an anterior and posterior chest tube was implanted for the patient.

In histopathology right upper lobe wedge resection, revealed severe suppurative necroinflammatory process associated with presence of a few partially degenerated fungal hyphae (both wide and irregular). Right lower lobe wedge resection showed extensive severe suppurative necroinflammatory process associated with presence of numerous fungal hyphae (both wide and irregular). Also vessel wall infiltrated by several fungal hyphae including both thin and broad fungal hyphae and calcium oxalate crystals accompanied by fungal hyphae was indicative of aspergillosis (Figure 2).

Hilar lymph node, biopsy revealed fibro-inflammatory process including foci of anthracotic pigment deposition. Fibro-adipose tissue showed bland looking gland like structures, patchy lymphocytic infiltration and presence of few large cells with cytopathology suggestive of CMV infection. Immunohistochemistry (IHC) was performed and confirmed the diagnosis of CMV pneumonia (Figure 3).

According to the invasive fungal infection with both Aspergillusand Mucoraceae species and also with the diagnosis of CMV disease, the liposomal amphotericin B, $5 \mathrm{mg} / \mathrm{kg} /$ day and intravenous gancyclovir $5 \mathrm{mg} / \mathrm{kg}$ every 12 hours were initiated. Also the imipenem with amikacin was continued for Klebsiella pneumoniae empyema. The patient signs and symptoms were improved and was afebrile. After discontinuation of amphotericin B and intravenous gancyclovir course, posaconazole oral suspension $200 \mathrm{mg}$ every 6 hours and valgancyclovir $900 \mathrm{mg}$ every 12 hours was started. The patient was discharged after one month with favorable clinical outcome.

\section{Discussion}

The possibility of SARS-CoV-2 co-infections with bacterial, fungal and also viral pathogens should not be neglected. Fungal co-infections among hospitalized patients with COVID-19 is a challenging issue. Few recent studies revealed cases of COVID-19 infection involving more than one species of aspergillus or concomitant two different fungal infections. Costache et al. reported a case of COVID-19 associated pulmonary aspergillosis with both Aspergillus fumigatus andAspergillus flavus with favorable outcome [2]. Johnson et al. 
reported a case of combined probable pulmonary aspergillosis and possible mucormycosis in a diabetic male with COVID-19 in the ICU who treated with liposomal amphotericin B [3].

Critical illness is an important risk factor for CMV reactivation due to immunosuppression. Although coinfections with CMV are frequent in the critically ill patients, but the impact on COVID-19 patients is unclear. Moniz et al. presented five case reports of CMV reactivation in COVID-19 patients admitted to the ICU with respiratory failure [4]. It has been well described that critical or severe illness can induce immune suppression. The most common laboratory finding in patients with COVID-19 is lymphopenia, which correlate to diseases severity. Recent findings revealed that disease severity is dominated by the decline in cell-mediated immunity especially the decrease in T cell counts [5]. On the other hand, number of iatrogenic factors including the usage of corticosteroids in the treatment of severe COVID-19 may predispose the patients to other infection [6]. In addition to ICU admission and mechanical ventilation, other entities including transfusions, sepsis, corticosteroids and acute respiratory distress syndrome have also been found to be associated with the risk of CMV reactivation [7].

In a minority of patients, bacterial and fungal co-infections can complicate the course of COVID-19 infection. In a retrospective study, the second most common respiratory pathogen detected from patients with COVID-19 was Klebsiella pneumoniae [8, 9]. Montrucchio et al. reported patients with COVID-19 related acute respiratory distress syndrome who developed invasive infections due to carbapenemase-producing Klebsiella pneumoniae [10]. In our patient, the culture of empyema and visceral pleural peel, confirmed the superimposed of Klebsiella pneumoniae infection on COVID-19.

Tissue invasion by a filamentous fungus through histopathological examination of biopsy, provides a diagnosis of proven invasive fungal infections and positive culture of Aspergillus from the specimen is required to make a proven diagnosis of invasive aspergillosis [11]. In our case, right upper lobe wedge resection and right lower lobe wedge resection revealed wide and irregular fungal hyphae indicative of mucormycosis. Also vessel wall infiltrated by several thin and also broad fungal hyphae. Calcium oxalate crystals accompanied by fungal hyphae was indicative of aspergillosis. Definitive diagnosis of CMV pneumonia is determined based on lung tissue samples by histopathologic testing and immunohistochemical analysis [12]. According to the data, our case is consistent with the proven CMV pneumonia.

According to the histopathology and IHC, our patient had pulmonary aspergillosis, mucormycosis, and CMV disease with Klebsiella pneumoniae infection. The combined risk factors including diabetes mellitus, recent corticosteroid treatment and also COVID-19 per se, contributed to concomitant infections in our patient which is a unique report.

\section{Conclusion}

Secondary infections or co-infections are identified between SARS-CoV-2 and other pathogens. This entity have become another serious threat in the treatment of patients with COVID-19 infection which should not be neglected.

\section{Author contributions}

P.T; M.PT; A.H; Z.A; A.M; M.R and M.MD acquired data, analyzed and interpreted the data. A.H wrote the first draft of the manuscript. All authors have read and approved the final manuscript.

\section{Disclosure of interest}

The authors declare that they have no competing interest.

\section{Ethical statement:}

This research was approved by the ethics committee of Shahid Beheshti University of Medical Sciences and written informed consent was obtained from the patient.

\section{Data availability statement:}


Data sharing is not applicable to this case report type article as no new data were created or analyzed in this study.

\section{Acknowledgments}

None.

\section{References}

1. Klein EY, Monteforte B, Gupta A, et al. The frequency of influenza and bacterial coinfection: a systematic review and meta-analysis. Influenza Other Respir Viruses 2016; 10: 394-403.

2. Costache C, Botan A, Mihaila RM, Colosi IA, Buksa SB, Chiorescu RM. Mixed Etiology COVID19 Associated Pulmonary Aspergillosis (CAPA) - A Case Report and Brief Review of the Literature. Journal of Fungi. 2021 Oct; 7(10):877.

3. Johnson AK, Ghazarian Z, Cendrowski KD, Persichino JG. Pulmonary aspergillosis and mucormycosis in a patient with COVID-19. Medical mycology case reports. 2021 Jun 1; 32:64-7.

4. Moniz P, Brito S, Póvoa P. SARS-CoV-2 and cytomegalovirus co-infections - a case series of critically ill patients. Journal of Clinical Medicine. 2021 Jan; 10(13):2792.

5. Jeannet R, Daix T, Formento R, Feuillard J, François B. Severe COVID-19 is associated with deep and sustained multifaceted cellular immunosuppression. Intensive care medicine. 2020 Sep; 46:1769-71.

6. Lionakis MS, Kontoyiannis DP. Glucocorticoids and invasive fungal infections. The Lancet. 2003 Nov $29 ; 362(9398): 1828-38$.

7. Osawa R, Singh N. Cytomegalovirus infection in critically ill patients: a systematic review. Critical Care. 2009 Jun; 13(3):1-0.

8. Rawson TM, Moore LS, Zhu N, Ranganathan N, Skolimowska K, Gilchrist M, Satta G, Cooke G, Holmes A. Bacterial and fungal coinfection in individuals with coronavirus: a rapid review to support COVID-19 antimicrobial prescribing. Clinical Infectious Diseases. 2020 Nov 1; 71(9):2459-68.

9. Zhu X, Ge Y, Wu T, Zhao K, Chen Y, Wu B, Zhu F, Zhu B, Cui L. Co-infection with respiratory pathogens among COVID-2019 cases. Virus Research. 2020 Aug 1; 285:198005.

10. Montrucchio G, Corcione S, Sales G, Curtoni A, De Rosa FG, Brazzi L. Carbapenem-resistant Klebsiella pneumoniae in ICU-admitted COVID-19 patients: Keep an eye on the ball. Journal of global antimicrobial resistance. 2020 Dec 1; 23:398-400.

11. De Pauw, B., Walsh, T.J., Donnelly, J.P., Stevens, D.A., Edwards, J.E., Calandra, T., Pappas, P.G., Maertens, J., Lortholary, O., Kauffman, C.A. and Denning, D.W., 2008. Revised definitions of invasive fungal disease from the European organization for research and treatment of cancer/invasive fungal infections cooperative group and the national institute of allergy and infectious diseases mycoses study group (EORTC/MSG) consensus group. Clinical infectious diseases, 46(12), pp.1813-1821.

12. Ljungman P, Griffiths P, Paya C. Definitions of cytomegalovirus infection and disease in transplant recipients. Clinical infectious diseases. 2002 Apr 15; 34(8):1094-7.

\section{Figure legends}

Figure 1. Multiple thick wall cavitary lesions in right upper lobe and ground glass opacities with complicated pneumothorax, pneumomediastinum and subcutaneous emphysema.

Figure 2 (A), Suppurative necrosis including several fungal hyphae, (B), vessel wall infiltrated by several fungal hyphae including both thin and broad fungal hyphae, (C), calcium oxalate crystals accompanied by fungal hyphae indicative of aspergillosis.

Figure 3 (A), Endothelial cells with cytoplasmic change of CMV, (B), IHC with CMV antibody confirming CMV cytopathic change.

\section{Hosted file}

Title page.docx available at https://authorea.com/users/453894/articles/551669-a-caseof-covid-19-complicated-by-klebsiella-pneumoniae-cytomegalovirus-aspergillus-and- 

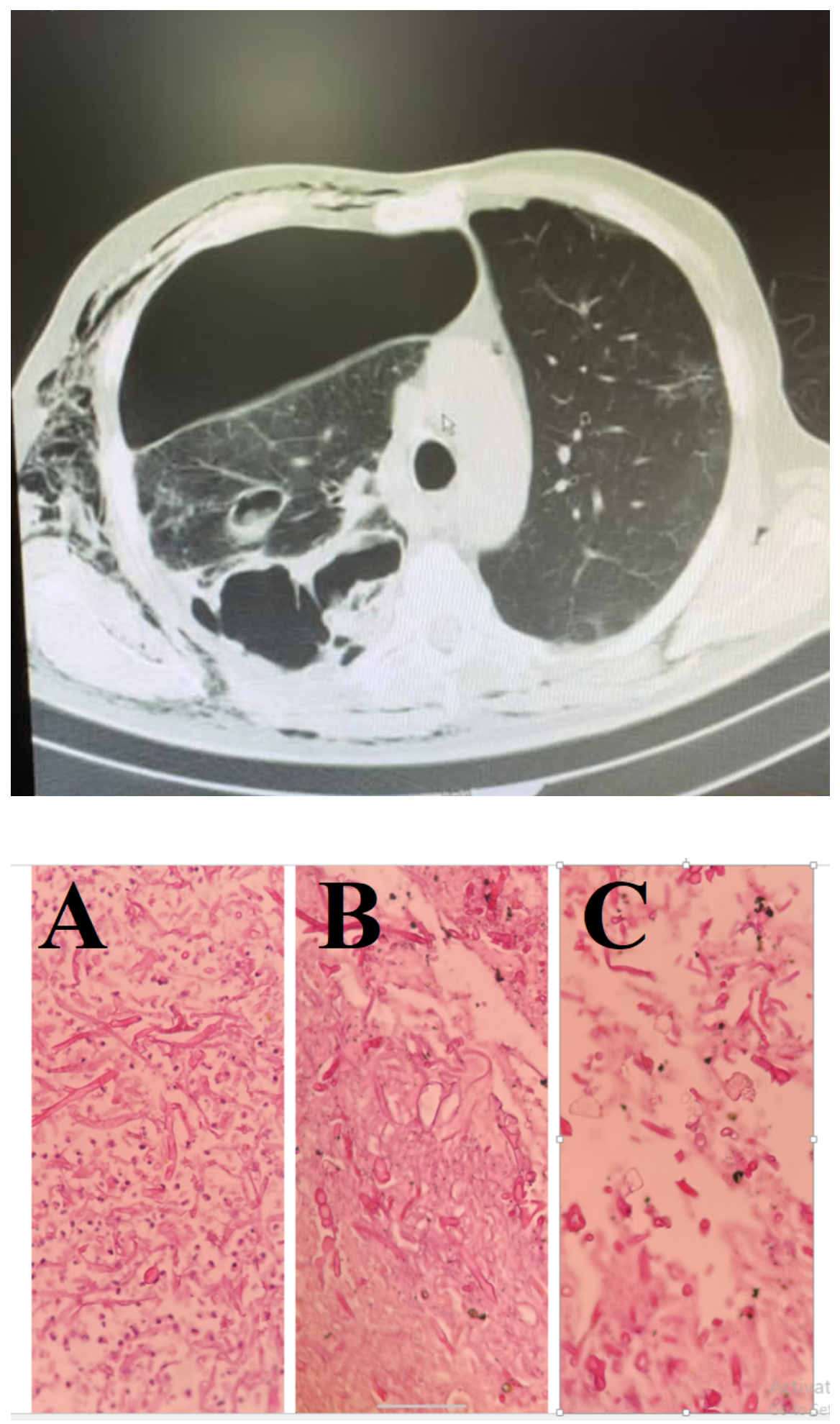


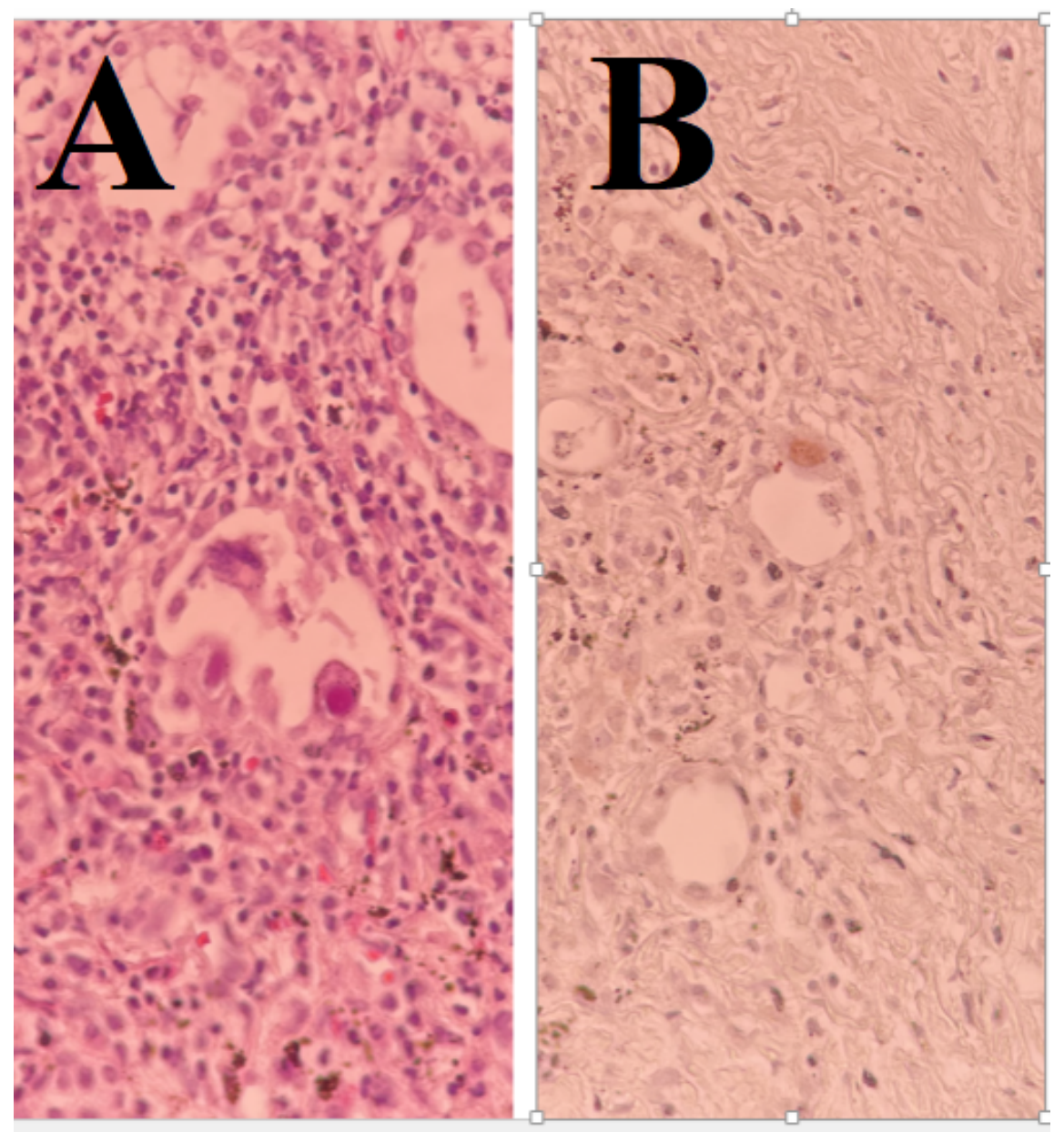

